\title{
Prospective Clinical Research of the Efficacy of Platelet-rich Plasma in the Outpatient-based Treatment of Rotator Cuff Tendinopathy
}

\author{
Ho-Won Lee, Kyung-Ho Choi, Jung-Youn Kim, Ik Yang ${ }^{1}$, Kyu-Cheol Noh \\ Departments of Orthopedic Surgery and ${ }^{1}$ Radiology, Hallym University Kangnam Sacred Heart Hospital, Seoul, Korea
}

Background: The purpose of this study is to compare the clinical outcomes of the control group and platelet-rich plasma (PRP) group among the patients who failed to respond to conservative treatment as outpatient-based therapy for rotator cuff tendinopathy, and to compare the clinical results of leukocyte-poor (LP) PRP and leukocyte-rich (LR) PRP.

Methods: Inclusion criteria are (1) over 18-year-old, (2) patients with rotator cuff tendinopathy, no rotator cuff tear by radiologic diagnosis (ultrasonography or magnetic resonance imaging) within the last 3 months, and (3) not effective to conservative treatment for more than 1 month. Of the final 60 subjects, 33 patients in the exercise treatment group and 27 patients in the PRP injection group (LP-PRP, 13; LR-PRP, 14) were included. Clinical evaluation was carried out by assessing the outcomes of treatment using the Numeric Rating Scale pain score, the American Shoulder and Elbow Society (ASES) score, and the Constant score at baseline and at 3 and 6 months after the procedure.

Results: There was a statistically significant difference in $\triangle \mathrm{ASES}_{3 \text { months }}\left(\mathrm{ASES}_{3 \text { months }}-\mathrm{ASES}_{\text {first }}\right)$ score between the control and PRP groups $(p=0.006)$. However, there was no statistical significance between LP-PRP and LR-PRP groups ( $p>0.05)$.

Conclusions: This study showed that PRP injection was more effective than exercise therapy for the first 3 months. However, there was no difference between the LP-PRP group and the LR-PRP group. Regardless of the type of PRP, clinical application of PRP injection in patients with rotator cuff tendinopathy seems to be effective in early treatment.

(Clin Shoulder Elbow 2019;22(2):61-69)

Key Words: Platelet-rich plasma; Rotator cuff; Tendinopathy; Exercise therapy

\section{Introduction}

Tendinopathy is one of the most common diseases and it accounts for $30 \%$ of all orthopedic musculoskeletal disorders. ${ }^{1)}$ The most common sites for tendinopathy include the elbow (the common extensor tendon and common flexor tendon, shoulder (the rotator cuff), ankle (the Achilles tendon), knee (the patellar tendon), and coxa (the gluteal tendon). Among them, rotator cuff tendinopathy is a major cause of shoulder pain and difficulties with activities of daily life, and its prevalence rate increases gradually with age, affecting more than $50 \%$ of the population aged 60 years. ${ }^{2)}$ In addition to a high prevalence rate, this dis- ease tends to recur frequently, and about $40 \%$ of patients with tendinopathy experience pain for more than a year. ${ }^{3)}$

It is known that rotator cuff tendinopathy is caused by a combined interaction of exogenous and intrinsic factors, and it causes degenerative changes in the rotator cuff.4) Degenerative changes in the rotator cuff cause acute inflammation and may progress to chronic inflammation and rotator cuff tears over time, eventually leading to full-thickness rotator cuff tears and cuff tear arthropathy. ${ }^{5}$

The first-line treatment for rotator cuff tendinopathy is conservative treatment, including rest, physical therapy, exercises, nonsteroidal anti-inflammatory drugs, and steroid injections. ${ }^{4,6)}$

Received December 10, 2018. Revised February 5, 2019. Accepted February 6, 2019.

Correspondence to: Kyu-Cheol Noh

Department of Orthopedic Surgery, Hallym University Kangnam Sacred Heart Hospital, 1 Singil-ro, Yeongdeungpo-gu, Seoul 07441, Korea Tel: +82-2-829-5165, Fax: +82-2-834-1728, E-mail: happynoh@gmail.com, ORCID: https://orcid.org/0000-0001-8738-2977

IRB approval: Hallym University Kangnam Sacred Heart Hospital (IRB No. 2014-07-9495).

Financial support: This research was supported by the 'Conditional Approval System of Health Technology' funded by the Ministry of Health and Welfare. Conflict of interests: None. 
Treatments focused on symptoms cannot prevent degenerative changes in the tendon and are not curative therapeutic modalities. In addition, although steroid injection therapy is the most commonly used treatment and can be effective in a short term of less than 3 months, its effect is not clear for longer periods." In addition, it inhibits collagen synthesis and also poses a risk of tendon rupture. ${ }^{8)}$ In recent years, platelet-rich plasma (PRP) has been introduced as a very safe and effective treatment for tendinopathy including rotator cuff tendinopathy. ${ }^{9}$

PRP has a higher platelet count compared to whole blood and has a variety of growth factors which help a wound heal. ${ }^{10,11)}$ Platelets include a variety of cytokines including growth factors such as the platelet-derived growth factor (PDGF) and transforming growth factor-beta (TGF- $\beta$ ), and these cytokines are involved in the recovery of injured tendons. ${ }^{12,13)}$ These substances play an important role in cell proliferation and differentiation, chemotaxis, and angiogenesis. Due to these various advantages of PRP, its application has been increasing and many positive study results have been reported. However, it is also true that some studies have reported that the effects of PRP are not clear. ${ }^{14,15)}$ There has been a study on the effect of PRP injection according to the concentration of white blood cells (WBC) among various components of PRP, and in recent studies, it has been reported that leukocyte-rich PRP (LR-PRP) causes inflammatory responses. ${ }^{16-18)}$ Although there have been a number of studies to compare the treatment by one method of PRP injection and the control group, few studies have compared clinical outcomes of two types of PRP in patients with rotator cuff tendinopathy.

Therefore, with respect to the outpatient-based treatment for patients with rotator cuff tendinopathy, this study aimed to compare the clinical outcomes of the exercise treatment group (control group) and the PRP injection group (PRP group) among patients who did not respond to conservative treatment, and to examine differences in clinical outcomes between the leukocyte-poor PRP (LP-PRP) and LR-PRP groups.

\section{Methods}

\section{Patients Enrollment}

The protocol for this study was approved by the Institutional Review Board (IRB) of Hallym University Kangnam Sacred Heart Hospital (IRB No. 2014-07-9495). This study was carried out as a prospective, quasi randomized clinical trial and participants were recruited from the patients who visited our institution. After providing an explanation of the purpose of the research, patients who voluntarily agreed to participate in the study were sequentially assigned to the PRP group or the control group. Among the patients who agreed to participate in the experiment, sequentially, odd-numbered patients were assigned to the PRP group and even-numbered patients were assigned to the control group. The patients assigned to the PRP group were again sequentially assigned to the LP-PRP group or LR-PRP group on an alternating basis. Then, an explanation about the costs, amounts of concentration, and levels of concentration of two types of PRPs were provided. In the case of patients diagnosed with tendinopathy in both the rotator cuffs, each of them was assigned as two participants when they wanted to have PRP injections in both sides.

The participants were selected based on the following criteria. The inclusion criteria were as follows: (1) patients aged 18 years or older; (2) patients who complain of shoulder pain and shoulder dysfunction, showing no rotator cuff tear but signs of rotator cuff tendinopathy on ultrasonography or magnetic resonance imaging; (3) patients who did not respond to a conservative treatment of 1 month or longer period; (4) females who have undergone surgical sterilization (hysterectomy or bilateral tubal sterilization), postmenopausal females, or fertile females who agreed to use medically acceptable methods of contraception (i.e., intrauterine contraceptive device, oral contraceptives, or spermicide barrier method); (5) patients who have not undergone rotator cuff surgery within the last 3 months; (6) patients who do not have septicemia or acute inflammatory disease (local infection at the treatment site); (7) patients who did not use steroid injection at the treatment site within the past one month or use systemic steroid within the past 2 weeks; (8) patients who voluntarily signed the written informed consent form and will comply with the procedures and requirements of clinical research.

The exclusion criteria were as follows: (1) patients diagnosed with a disease other than tendinopathy that causes pain in the treatment site, including arthritis and fracture, by radiological examination; (2) patients with hemodynamic instability; (3) patients with a blood disease inadequate for PRP extraction or injection therapy: patients with platelet dysfunction syndrome or a reduced platelet count (platelet count $<100,000 / \mu l$ ); (4) patients with a hemoglobin level of less than $10 \mathrm{~g} / \mathrm{dl}$ within the last one month; (5) patients who have had fever or an infectious disease within the last 2 weeks; (6) patients with a systemic disease such as rheumatoid arthritis and ankylosing spondylitis; (7) pregnant females and lactating females; (8) patients with a history of cancer (except for patients completely cured of cancer) or patients who have received cancer treatment within the past one year; (9) patients with an increased risk of bleeding due to the use of antiplatelet agents (the long-term, continuous use of major anticoagulants such as warfarin, heparin, and coumarin; except for patients taking with low-dose aspirin simply to prevent cardiovascular disease and patients who have undergone a washout period for major anticoagulants); (10) patients who have been habitually taking psychotropic substances or narcotic analgesics that can affect pain sensation for 3 months or more; (11) patients to whom other study drugs have been administered within 30 days before participating in a study related to the conditional approval system of health technology. 
In this study, among 70 patients who met the inclusion criteria, 64 patients were finally enrolled, excluding 6 patients according to the exclusion criteria, including surgery and steroid injection therapy. Of 64 patients, 34 were assigned to the exercise treatment group (control group) and 30 patients were assigned to the PRP injection group (PRP group). Excluding 1 participant lost to follow-up from the control group and 3 participants (2 participants in the LP-PRP group, 1 participant in the LR-PRP group; lost to follow-up and taking steroids) from the PRP group, a total of 33 patients in the control group and 27 patients in the PRP group were included in the final analysis (Fig. 1).

\section{Platelet-rich Plasma Preparation}

LP-PRP $4 \mathrm{ml}$ was prepared using the $\mathrm{ACP}^{\mathrm{TM}}$ kit (Arthrex, Naples, FL, USA) after collecting $15 \mathrm{ml}$ of blood. One double syringe containing coagulant was connected with a butterfly needle to collect $15 \mathrm{ml}$ of blood. Centrifugation was performed at 1,500 rpm for 5 minutes. The outer plunger of the double syringe was slowly pressed while the inner plunger was slowly pulled to separate PRP appropriately. Out of the PRP (about 4 $\mathrm{ml}), 1.5 \mathrm{ml}$ obtained by a double syringe by the centrifugation method was injected in the rotator cuff under ultrasound guidance. Using the GPS ${ }^{\text {TM }}$ III kit (Biomet, Warsaw, IN, USA), $6 \mathrm{ml}$ of LR-PRP was obtained after collecting $60 \mathrm{ml}$ of blood. One GPS kit was opened and $6 \mathrm{ml}$ of anticoagulant was put in each $60 \mathrm{ml}$ syringe. The $60 \mathrm{ml}$ syringe containing anticoagulant was connected with the butterfly needle, and $54 \mathrm{ml}$ of blood was collected. Then, centrifugation was performed at 3,200 rpm for about 15 minutes. Out of PRP (about $6 \mathrm{ml}$ ), $1.5 \mathrm{ml}$ obtained from the centrifuge vessel was injected in the rotator cuff under

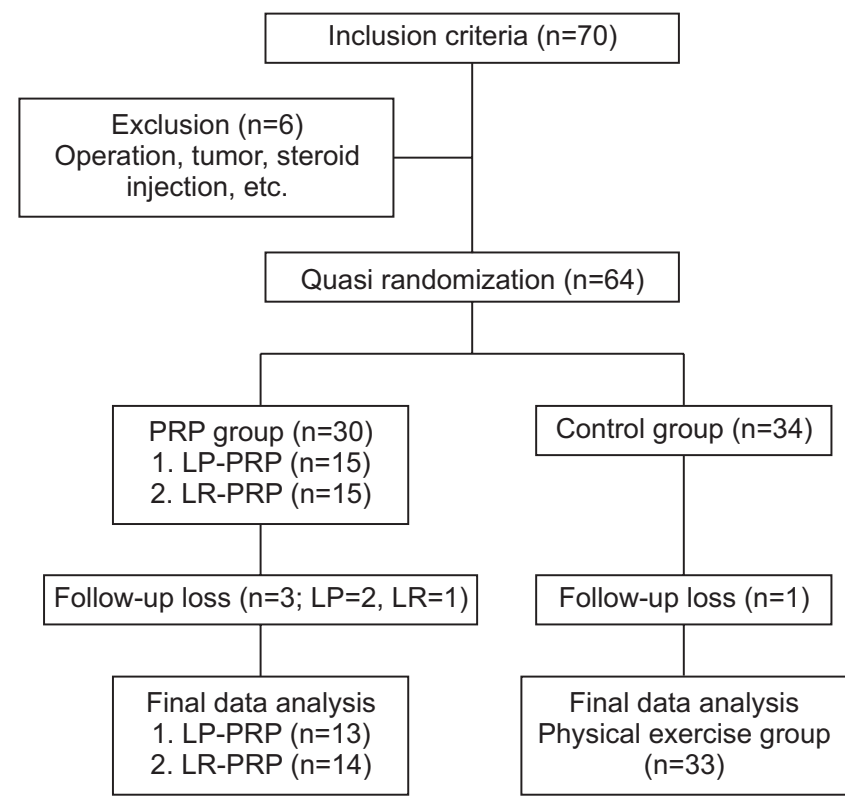

Fig. 1. Flow chart of patients in the study.

PRP: platelet-rich plasma, LP: leukocyte-poor, LR: leukocyte-rich. ultrasound guidance.

\section{Ultrasonographic Elvaluation and Platelet-rich Plasma Injection}

The PRP group and the control group were placed in an upright position, and the thickness of the supraspinatus was measured by placing an ultrasonic detector on the supraspinatus in a modified crass position. The longest area vertically from the glomerular tuberosity to the supraspinatus was measured. This measurement was performed both in the PRP group and the control group, and a comparison was made at baseline and at the final follow-up after 6 months. This evaluation was conducted by a professor in the department of radiology (Fig. 2).

After the initial ultrasound evaluation, the participants in the PRP group were placed in an upright position and the maximum external rotation of the upper arm with a degenerative rotator cuff tear lesion was performed. Using ultrasonography, the cross section of the long head of biceps tendon was identified, and by moving to the proximal region while maintaining the cross section, the ultrasonic detector was placed on the supraspinatus muscle. Using a needle, $1.5 \mathrm{ml}$ of autologous PRP was injected into the degenerative area of the supraspinous muscle in a horizontal direction under ultrasound guidance (Fig. 2). When PRP was injected into the lesion areas of both rotator cuffs, $1.5 \mathrm{ml}$ was injected into the lesion of each side. In this case, after PRP was injected into one lesion area, it was injected into another lesion area in the same manner. The procedure was performed by an orthopedic surgeon doing a 2-year fellowship in the field of shoulder and elbow surgery.

\section{Exercise Treatment}

Exercise treatment (rotator cuff strengthening exercise) was continuously performed using known exercises related to rotator cuff disease in both control and PRP groups. However, in

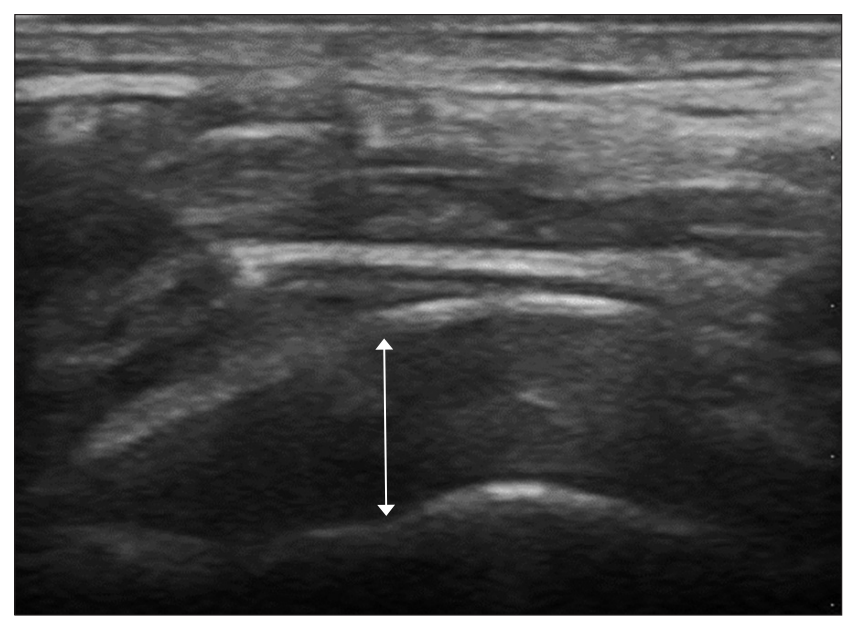

Fig. 2. Ultrasound-guided platelet-rich plasma injection and measurement of tendon thickness. 
the PRP group, PRP injection treatment was performed before exercise treatment. Regarding exercise treatment, both the control group and PRP group were educated about the detailed techniques for both shoulder stretching and strengthening exercises under the supervision of a professor in the department of rehabilitation medicine. Patients were requested to perform exercises for 20 minutes a day for at least four days a week. Passive range of motion (ROM) exercises and shoulder stretching exercises were performed for the first week, and pain relief and restoration of ROM were examined. Then, stabilization exercise and isometric exercise are performed for 3 weeks through education, and muscle strengthening exercises using dumbbells and rubber bands were performed until the final follow-up 6 months after the treatment.

\section{Clinical Outcome Assessment}

Clinical outcome assessments were carried out using the Numeric Rating Scale (NRS) for pain, the American Shoulder and Elbow Society (ASES) score, and the Constant score at baseline and at 3 months and 6 months after the intervention. ${ }^{19,20)}$ The assessment by the NRS for pain is performed by the patient's subjective self-assessment of pain intensity on a scale of 0 to 10 , and the ASES score consists of assessments for subdivided categories of pain and activities of daily living. The Constant score is divided into the subjective evaluation of pain intensity and daily activities (35 points) and objective assessment of ROM and mus- cular strength (65 points).

\section{Statistical Analysis}

Using G-Power 3.1.5, based on the effect size of 0.80, significance level of 0.05 , and power of 0.80 , the sample size was determined to be a total of 60 patients (30 patients per group) considering the dropout rate of at least $10 \%$. In this study, participants consisted of 30 patients in the PRP group and 34 patients in the control group, and excluding dropouts, 27 patients in the PRP group and 33 patients in the control group were included in the final analysis.

For data analysis, continuous variables were presented as mean \pm standard deviation, and categorical variables were presented as the number of times. The chi-square test or Fisher exact test was performed for categorical data. In addition, an independent two-sample t-test was conducted to determine if there was a significant difference in blood information between groups. The Mann-Whitney test was used to determine if there was a difference in growth factors between groups. In order to compare the differences between the PRP group and the control group in clinical scores, the significance of changes due to the intervention was verified by covariance analysis (ANCOVA), and the Bonferroni post-hoc test was performed to examine differences in the follow-up care among the LP-PRP, LR-PRP, and control groups. All statistical analyses were performed using IBM SPSS ver. 20.0 (IBM Corp. Armonk, NY, USA). The level of statis-

Table 1. Demographic Data of PRP and Control Groups at Baseline

\begin{tabular}{|c|c|c|c|c|}
\hline \multirow{2}{*}{ Variable } & \multicolumn{2}{|c|}{ PRP group $(n=27)$} & \multirow{2}{*}{ Control group $(n=33)$} & \multirow{2}{*}{$p$-value } \\
\hline & LP-PRP $(n=13)$ & LR-PRP $(n=14)$ & & \\
\hline Sex & & & & 0.263 \\
\hline Male & 5 & 6 & 7 & \\
\hline Female & 8 & 8 & 26 & \\
\hline Age (yr) & $51.31 \pm 7.25$ & $53.00 \pm 9.52$ & $57.58 \pm 6.38$ & $0.021^{*}$ \\
\hline Height (cm) & $161.92 \pm 10.17$ & $162.71 \pm 10.01$ & $160.21 \pm 7.83$ & 0.822 \\
\hline Weight (kg) & $66.00 \pm 11.90$ & $66.43 \pm 11.04$ & $63.30 \pm 8.56$ & 0.527 \\
\hline Dominant hand & & & & $0.002^{\star}$ \\
\hline Right & 12 & 13 & 17 & \\
\hline Left & 1 & 1 & 16 & \\
\hline Involved site & & & & $<0.001^{\star}$ \\
\hline Right & 6 & 7 & 30 & \\
\hline Left & 7 & 7 & 3 & \\
\hline DM & & & & $>0.999$ \\
\hline No & 11 & 12 & 29 & \\
\hline Yes & 2 & 2 & 4 & \\
\hline
\end{tabular}

Values are presented as number only or mean \pm standard deviation.

PRP: platelet-rich plasma, LP: leukocyte poor, LR: leukocyte rich, DM: diabetes mellitus.

${ }^{\star}$ Significant difference compared with baseline $(p<0.05)$. 
tical significance was set at $p<0.05$.

\section{Results}

In this study, no specific side effects occurred during the treatment period. Especially, infections and neurological problems that may occur during injection therapy did not occur.

In terms of demographic data, there was no statistically significant differences between the PRP group and the control group in sex, diabetes diagnosis status, height, and weight $(p<0.05)$, but the two groups showed statistically significant differences in the dominant hand $(p=0.002)$, involved site $(p<0.001)$, and age $(p=0.021)$ (Table 1). In the PRP group, a comparison between the LR-PRP group and the LP-PRP group showed that WBC count $(p<0.001)$ and platelet count $(p=0.005)$ were statistically significantly higher in the LR-PRP group than in the LP-PRP group. However, the two groups did not show any statistically significant differences in the levels of cytokines, such as PDGF, TGF- $\beta$, vascular endothelial growth factor, insulin-like growth factor 1 beta, and interleukin 8 ( $p>0.05$ ) (Table 2).

The measurement of changes in the thickness of the tendon by ultrasonography showed that the mean value of the control group decreased from $1.10 \mathrm{~cm}$ at baseline to $1.08 \mathrm{~cm}$ at 6 months, whereas the PRP group showed an increase from 0.99 at baseline to $1.05 \mathrm{~cm}$ at 6 months. A higher increase in the thickness of the tendon in the PRP group compared to the control group was confirmed by ultrasonography, but the difference was minimal and not statistically significant.

When differences between the PRP group and the control group (exercise treatment group) were examined by adjusting age and baseline values, there was a significant difference in the ASES score at 3 months $(p=0.001)$. The ASES score increased from 60.21 points at baseline to 66.12 points at 3 months in the control group, and it increased from 57.93 points at baseline to 73.89 points at 3 months in the PRP group. At 6 months, the
ASES score was higher in the PRP group (80.3 points) than in the control group (75.88 points), but there was no significant difference between the two groups at 6 months. In the Constant score and NRS score, there was no statistically significant difference between the two groups (Fig. 3).

The analysis of clinical scores showed that there were statistically significant differences in $\triangle \mathrm{ASES}_{3 \text { months }}\left(\mathrm{ASES}_{3 \text { months }}-\mathrm{ASES}_{\text {first }}\right)$ among the control and PRP (LP-PRP, LR-PRP) groups $(p=0.006)$, and the analysis of differences between subgroups showed that there were also statistically significant differences between the LP-PRP and control groups $(p=0.011)$ and between the LRPRP and control groups $(p=0.030)$. However, there was no statistically significant difference between the LP-PRP and LRPRP groups in $\triangle \mathrm{ASES}_{6 \text { months, }} \Delta$ Constant score, and $\triangle \mathrm{NRS}$ score $(p>0.05)$ (Table 3, 4).

Table 2. Laboratory Data of PRPs Used

\begin{tabular}{lccc}
\hline \multirow{2}{*}{ Variable } & \multicolumn{2}{c}{ PRP group } & \multirow{2}{*}{$p$-value } \\
\cline { 2 - 3 } & LP-PRP & LR-PRP & \\
\hline WBC $\left(\times 10^{3} / \mu \mathrm{l}\right)$ & $1.46 \pm 1.30$ & $20.14 \pm 7.27$ & $<0.001^{\star}$ \\
\hline Platelet $\left(\times 10^{3} / \mu \mathrm{l}\right)$ & $335.46 \pm 88.24$ & $766.64 \pm 481.13$ & $0.005^{\star}$ \\
\hline PDGF $(\mathrm{pg} / \mathrm{ml})$ & $4.67 \pm 4.43$ & $7.63 \pm 3.48$ & 0.275 \\
TGF- $\beta(\mathrm{pg} / \mathrm{ml})$ & $1,231.72 \pm 1,395.27$ & $1,185.28 \pm 1,335.50$ & 0.682 \\
\hline VEGF $(\mathrm{pg} / \mathrm{ml})$ & $23.06 \pm 32.92$ & $26.65 \pm 45.31$ & 0.287 \\
\hline IGF-1 $(\mathrm{pg} / \mathrm{ml})$ & $513.33 \pm 171.98$ & $626.03 \pm 425.12$ & 0.852 \\
\hline IL-1 $\beta(\mathrm{pg} / \mathrm{ml})$ & $10.10 \pm 7.79$ & $10.41 \pm 7.29$ & 0.627 \\
\hline IL-8 $(\mathrm{pg} / \mathrm{ml})$ & $3,783.34 \pm 5,180.87$ & $3,638.45 \pm 4,984.66$ & 0.732 \\
\hline
\end{tabular}

Values are presented as mean \pm standard deviation.

PRP: platelet-rich plasma, LP: leukocyte poor, LR: leukocyte rich, WBC: white blood cell, PDGF: platelet-derived growth factor, TGF- $\beta$ : transforming growth factor beta, VEGF: vascular endothelial growth factor, IGF-1: insulinlike growth factor 1 , IL-1 $\beta$ : interleukin 1 beta, IL-8: interleukin 8.

${ }^{*}$ Significant difference compared with baseline $(p<0.05)$.
A

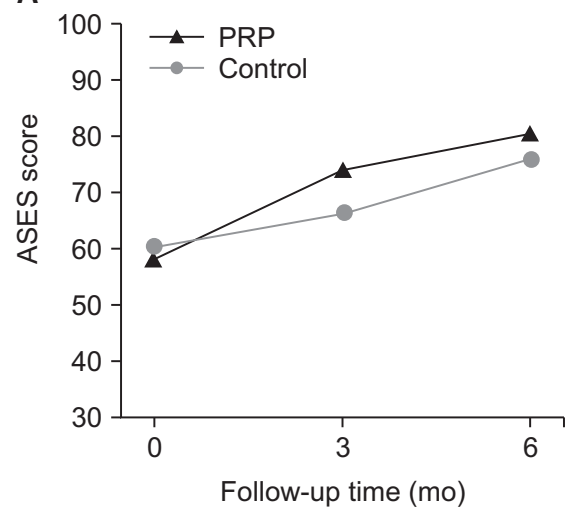

B

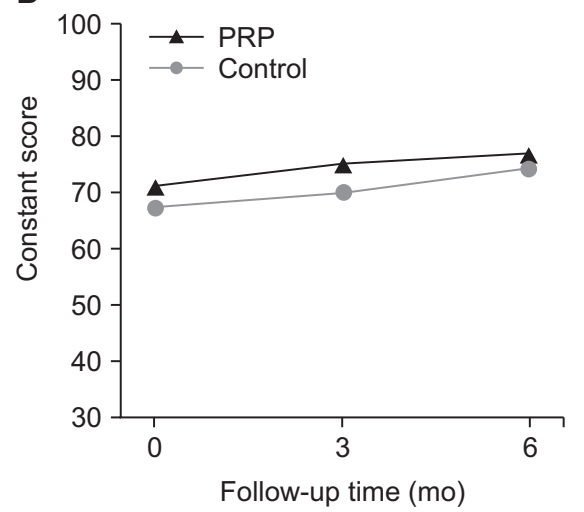

C

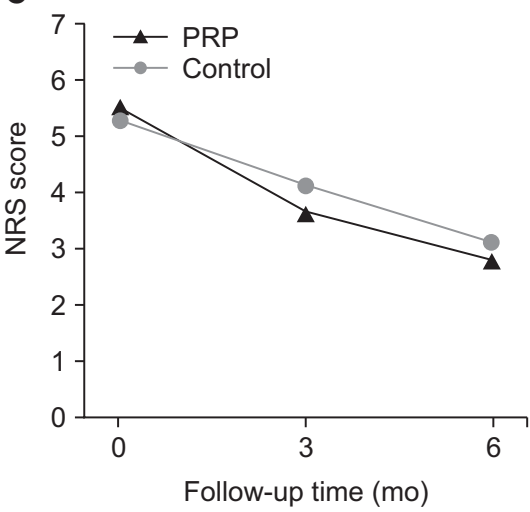

Fig. 3. Changes in the American Shoulder and Elbow Society (ASES) score (A), Constant score (B), and Numeric Rating Scale (NRS) score (C) over time. PRP: platelet-rich plasma. 
Table 3. Comparison of Clinical Score Difference between PRP and Control Groups

\begin{tabular}{|c|c|c|c|c|}
\hline \multirow{2}{*}{ Variable } & \multicolumn{2}{|c|}{ PRP group } & \multirow{2}{*}{ Control group } & \multirow{2}{*}{$p$-value ${ }^{\dagger}$} \\
\hline & LP-PRP & LR-PRP & & \\
\hline \multicolumn{5}{|l|}{ ASES score } \\
\hline$\Delta \mathrm{ASES}_{3 \text { months }}$ & $10.92 \pm 11.63$ & $20.64 \pm 15.40$ & $5.91 \pm 7.64$ & $0.006^{*}$ \\
\hline$\Delta \mathrm{ASES}_{6 \text { months }}$ & $12.85 \pm 14.29$ & $31.21 \pm 14.23$ & $15.67 \pm 10.14$ & 0.054 \\
\hline \multicolumn{5}{|l|}{ Constant score } \\
\hline$\Delta$ Constant $_{3 \text { months }}$ & $1.69 \pm 6.91$ & $5.57 \pm 6.95$ & $2.55 \pm 6.15$ & 0.227 \\
\hline$\Delta$ Constant $_{6 \text { months }}$ & $1.62 \pm 7.25$ & $9.21 \pm 5.78$ & $6.64 \pm 6.87$ & 0.226 \\
\hline \multicolumn{5}{|l|}{ NRS score } \\
\hline$\Delta \mathrm{NRS}_{3 \text { months }}$ & $-0.85 \pm 1.41$ & $-2.79 \pm 2.26$ & $-1.15 \pm 1.28$ & 0.364 \\
\hline$\Delta \mathrm{NRS}_{6 \text { months }}$ & $-1.00 \pm 1.68$ & $-4.29 \pm 2.16$ & $-2.18 \pm 1.33$ & 0.125 \\
\hline
\end{tabular}

Values are presented as mean \pm standard deviation.

$\triangle \mathrm{ASES}_{\text {months }}$ is calculated as $\mathrm{ASES}_{\text {months }}-\mathrm{ASES}_{\text {first }} ; \Delta$ Constant $_{\text {months }}$ is calculated as Constant months - Constant $_{\text {first }} ; \Delta \mathrm{NRS}_{3 \text { months }}$ is calculated as $\mathrm{NRS}_{\text {months }}-\mathrm{NRS}_{\text {first }}$. PRP: platelet-rich plasma, LP: leukocyte poor, LR: leukocyte rich, ASES: American Shoulder and Elbow Society, NRS: Numeral Rating Scale.

${ }^{*}$ Significant difference $(p<0.05)$. ${ }^{\dagger}$ Analysis of covariance (ANCOVA) adjusted $p$-value with age.

Table 4. Subgroup Analysis between PRP and Control Groups of $\triangle \mathrm{ASES}_{3 \text { months }}$ LP-PRP vs. LR-PRP LP-PRP vs. Control LR-PRP vs. Control

\begin{tabular}{|c|c|c|c|}
\hline$p$-value ${ }^{\dagger}$ & $>0.999$ & $0.011^{*}$ & $0.030^{*}$ \\
\hline
\end{tabular}

$\triangle \mathrm{ASES}_{\text {months }}$ is calculated as $\mathrm{ASES}_{\text {months }}-\mathrm{ASES}_{\text {first }}$.

PRP: platelet-rich plasma, ASES: American Shoulder and Elbow Society, LP: leukocyte poor, LR: leukocyte rich.

${ }^{*}$ Significant difference $(p<0.05)$. ${ }^{\dagger}$ Bonferroni post-hoc test by group.

\section{Discussion}

The most important result in this study was that there was a statistically significant improvement in the clinical score (ASES score) at 3 months in the PRP group (LP-PRP, LR-PRP) compared to the control group. At the final follow-up (6 months), ASES scores compared to baseline values were definitely higher in the PRP group than in the control group, but there were no statistically significant differences. The clinical scores of the PRP group were generally better than the control group. However, additional analysis of the subgroups in the PRP group showed no statistically significant differences between LP-PRP and LR-PRP groups.

Currently, clinically used PRP is an ideal autologous biological product containing various growth factors and a large amount of active substances such as cytokines. PRP has a high concentration of PDGFs to help heal the tendon through effects such as cell proliferation, angiogenesis and collagen synthesis. ${ }^{21)}$ PRP injection is used in various areas such as the elbow (common extensor tendon, common flexor tendon), shoulder (rotator cuff), ankle (Achilles ligament), and knee (patellar tendon). In rotator cuff disease, it has been demonstrated that PRP injection is more stable and efficacious than the most commonly used steroid injection therapy. ${ }^{22)}$ In light of these facts, it was judged that PRP injection may be effective in patients with rotator cuff disease.

Previous studies of PRP injection reported different results regarding symptoms and clinical improvements. ${ }^{23-27)}$ Moraes et al. ${ }^{28)}$ presented a comparative analysis of 19 randomized control trials in the Cochrane review. Their study, which included 1,088 patients, compared the PRP group with the control group (placebo, autologous whole bloods, dry needling, not PRP), reported that there was no sufficient evidence that the use of PRP was effective, and suggested that the standardization of PRP preparation methods was required. ${ }^{28)}$ However, a recent systematic review and meta-analysis compared the effects of LP-PRP and LR-PRP in terms of function and pain intensity, and reported that LR-PRP resulted in better clinical outcomes in patients with tendinopathy. ${ }^{29)}$ Although there have been some debates over pain relief and clinical outcomes at the final follow-up, in an in vitro study, we found that the growth factors of LP-PRP enhance human fibroblast proliferation. ${ }^{30)}$ In addition, in a pilot study, it was shown that more growth factors were secreted in LR-PRP compared to LP-PRP. Therefore, we predicted that PRP injection therapy would result in a greater improvement in clinical scores compared to the control group treated only with exercise therapy in an in vivo study, and hypothesized that LR-PRP would achieve better clinical outcomes than LP-PRP. In this study, the PRP injection group showed a statistically significantly greater improvement in the ASES score compared to the control group. This result is consistent with the findings of recent studies. Jo et al. ${ }^{27)}$ reported that allogenic PRP had significantly higher effects on the recovery of shoulder functions and pain relief compared to steroid injection at 6 months after the treatment for inflammatory conditions such as rotator cuff disease or tendinopathy. In addition, according to a randomized controlled 1-year followup study of patients with chronic rotator cuff tendinopathy, a 
comparison between the exercise group and PRP group showed that there was no statistically significant difference between the two groups. However, the study also reported that there was a statistically significant improvement in the Western Ontario Rotator Cuff Index, Shoulder Pain and Disability Index, and visual analogue scale score in the PRP injection group. ${ }^{23)}$ In a previous study which compared subacromial ACP injection and cortisone injection treatments, although there was no statistically significant difference between ACP injection and cortisone injection groups at the final follow-up 6 months after the treatment, there was a significant difference between the two groups at 6 weeks and 12 weeks. ${ }^{31}$ In the study, 3 LP-PRP injections were performed at intervals of 7 days. Unlike previous studies, PRP injection was performed only once in this study. Even with one injection, there was a significant difference between the PRP and control groups at 3 months. However, although there was a significant increase in ASES scores in the PRP injection group at 6 months, there were no statistically significant differences between the two groups $(p=0.054)$. At the final follow-up, it was clearly confirmed that PRP injection group showed better ASES scores. It is presumed that if the sample size or the number of PRP injections had been larger, it would have been possible to reveal statistical differences at the final follow-up.

This study was conducted using two main types of PRP. The first type is PRP obtained from the plasma layer. This is obtained by extracting platelets from the remaining plasma layer after eliminating red blood cells (RBC) and WBC as much as possible during the preparation process. In this type of PRP, since the RBC count and WBC count are low, the platelet count is also low. In our ACP kit, the PRP concentration was approximately 1.3 times that of whole blood, with an average of $372.9 \times 10^{3} /$ $\mu \mathrm{l}$, and the concentrations of various growth factors were also low. ${ }^{32)}$ The role of WBCs in the PRP has not yet been clarified. However, some studies found that WBCs enhance catabolic signaling, and some studies reported that LR-PRP is more likely to cause acute inflammatory responses. ${ }^{33,34)}$ In addition, it was previously reported that LP-PRP induces a greater increase in collagen synthesis and a greater reduction in inflammationinducing cytokines compared with LR-PRP. ${ }^{35)}$ The second type of PRP is a PRP product obtained from the buffy coat layer. It is a mixture of platelets and leukocyte-rich plasma, and platelets are obtained from both the plasma layer and the cell layer. In general, the platelet count is much higher and is approximately 3 to 8 times as high as the baseline value. ${ }^{36,37}$ In this study, we used the GPS III kit. Various studies using high-concentration LR-PRP have reported good results in meta-analysis. ${ }^{38)}$ Although there have been relatively fewer studies using ACP, a previous study reported statistically significant results compared to cortisone injection therapy until 3 months after the treatment, and another previous study reported that there was a greater improvement in the score of the pain assessment scale compared to dry needling until 6 months after the treatment, but did not show any statistically significant differences. ${ }^{39)}$ Yan et al. ${ }^{40)}$ compared LP-PRP with LR-PRP using the Achilles tendinopathy animal model, found that LP-PRP promotes tendon healing more efficiently through anabolic effects in patients with chronic tendinopathy in an in vivo study, and suggested that LP-PRP may be a better option in the clinical application for chronic tendinopathy. Most of the previous studies compared only one type of PRP to the control group, and thus they did not reveal which type between LPPRP and LR-PRP show better clinical outcomes in patients with rotator cuff disease. In contrast, this study compared clinical outcomes of LP-PRP and LR-PRP treatments in patients with rotator cuff disease. There was a statistically significant difference in the changes in clinical scores ( $\triangle$ ASES) at 3 months between the PRP group and the control group $(p=0.011,0.030)$, but there was no significant difference at 6 months $(p=0.141)$. In the subgroup analysis for the PRP group, the mean ASES score was higher in the LR-PRP group than in the LP-PRP group, but there were no statistically significant results. According to Lim et al., ${ }^{41)}$ TGF- $\beta$ levels in PRP play an important role in the recovery of the tendon and are associated with clinical scores. Unlike previous pilot data, there was no significant difference in TGF- $\beta$ levels between the ACP and GPS group in this study. These results suggest that there is a possibility that the differences in clinical scores between the two groups may have not been identified. Moreover, differences in cytokines such as TGF- $\beta$ may be associated with better clinical outcomes rather than simply products with a high WBC count and platelet count. In addition, since the analysis of PRP was conducted by divided the PRP group into two groups unlike other studies, it is possible that since the number of patients in each group was smaller, statistically significant differences in clinical results were not derived.

This study has some limitations. First, statistical analysis did not show sufficiently satisfactory results because of a small sample size. Since the $\mathrm{N}$ value was small, the standard deviation became large, so it was not possible to determine the statistical significance of many values. Second, since this study was a quasi randonmized clinical trial design, there is a possibility of the selection bias. Third, there is a possibility of the effect of the exercise therapy related to rotator cuff tendinopathy performed after PRP injection. In the control group, only exercise therapy was performed, so it is thought that if dry needling, saline, or steroid injection process is included in future studies, better study outcomes can be obtained. Finally, only one PRP injection was carried out in this study. Although there are no definite criteria for the intervals between PRP injections, Rha et al. ${ }^{24)}$ performed PRP injection and dry needling at intervals of 4 weeks in patients with rotator cuff disease, and reported that the PRP injection group showed better clinical outcomes than the dry needling group at 6 months. In future clinical applications, if the time for the completion of treatment is assumed to be 6 months (24 
weeks), additional PRP injections at appropriate time points may provide better results, which needs to be clarified in a future study. This study has the following strengths. First, the lidocaineinduced pain relief was excluded by not performing lidocaine injection. Second, the clinical effects of LP-PRP and LR-PRP on rotator cuff disease were compared.

\section{Conclusion}

In this study, it was found that the PRP injection group showed better clinical outcomes than the control group for the first 3 months. However, there was no difference between the LP-PRP and LR-PRP groups, and it was shown that the effect of PRP injection was not always influenced by leukocyte and platelet counts. The study results indicate that regardless of the PRP type, clinical application of PRP injection is effective in early treatment in patients with rotator cuff disease who do not respond to conservative treatment.

\section{Acknowledgements}

The authors would like to express their gratitude to Ms. Su-Jin Jung for her technical assistance and contributions to the statistical analysis.

\section{References}

1. Kaux JF, Forthomme B, Goff CL, Crielaard JM, Croisier JL. Current opinions on tendinopathy. J Sports Sci Med. 2011;10(2): 238-53.

2. Seitz AL, McClure PW, Finucane S, Boardman ND 3rd, Michener LA. Mechanisms of rotator cuff tendinopathy: intrinsic, extrinsic, or both? Clin Biomech (Bristol, Avon). 2011;26(1):112. doi: 10.1016/j.clinbiomech.2010.08.001.

3. Lewis JS. Rotator cuff tendinopathy/subacromial impingement syndrome: is it time for a new method of assessment? $\mathrm{Br} J$ Sports Med. 2009;43(4):259-64. doi: 10.1136/bjsm.2008. 052183.

4. Hermans J, Luime JJ, Meuffels DE, Reijman M, Simel DL, Bierma-Zeinstra SM. Does this patient with shoulder pain have rotator cuff disease?: the rational clinical examination systematic review. JAMA. 2013;310(8):837-47. doi: 10.1001/jama. 2013.276187.

5. Jo CH, Shin WH, Park JW, Shin JS, Kim JE. Degree of tendon degeneration and stage of rotator cuff disease. Knee Surg Sports Traumatol Arthrosc. 2017;25(7):2100-8. doi: 10.1007/ s00167-016-4376-7.

6. Mitchell C, Adebajo A, Hay E, Carr A. Shoulder pain: diagnosis and management in primary care. BMJ. 2005;331(7525): 1124-8. doi: 10.1136/bmj.331.7525.1124.

7. Coombes BK, Bisset L, Vicenzino B. Efficacy and safety of cor- ticosteroid injections and other injections for management of tendinopathy: a systematic review of randomised controlled trials. Lancet. 2010;376(9754):1751-67. doi: 10.1016/S01406736(10)61160-9.

8. Browning DG, Desai MM. Rotator cuff injuries and treatment. Prim Care. 2004;31(4):807-29. doi: 10.1016/j.pop.2004.08.004.

9. Engebretsen L, Steffen K, Alsousou J, et al. IOC consensus paper on the use of platelet-rich plasma in sports medicine. $\mathrm{Br}$ J Sports Med. 2010;44(15):1072-81. doi: 10.1136/bjsm.2010. 079822.

10. Alsousou J, Thompson M, Hulley P, Noble A, Willett K. The biology of platelet-rich plasma and its application in trauma and orthopaedic surgery: a review of the literature. J Bone Joint Surg Br. 2009;91(8):987-96. doi: 10.1302/0301-620X. 91B8.22546.

11. Pietrzak WS, Eppley BL. Platelet rich plasma: biology and new technology. J Craniofac Surg. 2005;16(6):1043-54. doi: 10.1097/01.scs.0000186454.07097.bf.

12. Hsu C, Chang J. Clinical implications of growth factors in flexor tendon wound healing. J Hand Surg Am. 2004;29(4):551-63. doi: 10.1016/j.jhsa.2004.04.020.

13. Zhang J, Middleton KK, Fu FH, Im HJ, Wang JH. HGF mediates the anti-inflammatory effects of PRP on injured tendons. PLoS One. 2013;8(6):e67303. doi: 10.1371/journal.pone.0067303.

14. de Vos RJ, Weir A, van Schie HT, et al. Platelet-rich plasma injection for chronic Achilles tendinopathy: a randomized controlled trial. JAMA. 2010;303(2):144-9. doi: 10.1001/ jama.2009.1986.

15. Nguyen RT, Borg-Stein J, Mclnnis K. Applications of plateletrich plasma in musculoskeletal and sports medicine: an evidence-based approach. PM R. 2011;3(3):226-50. doi: 10.1016/j.pmrj.2010.11.007.

16. Hudgens JL, Sugg KB, Grekin JA, Gumucio JP, Bedi A, Mendias $\mathrm{CL}$. Platelet-rich plasma activates proinflammatory signaling pathways and induces oxidative stress in tendon fibroblasts. Am J Sports Med. 2016;44(8):1931-40. doi: 10.1177/ 0363546516637176.

17. Zhang L, Chen S, Chang P, et al. Harmful effects of leukocyterich platelet-rich plasma on rabbit tendon stem cells in vitro. Am J Sports Med. 2016;44(8):1941-51. doi: 10.1177/ 0363546516644718.

18. Zhou Y, Zhang J, Wu H, Hogan MV, Wang JH. The differential effects of leukocyte-containing and pure platelet-rich plasma (PRP) on tendon stem/progenitor cells - implications of PRP application for the clinical treatment of tendon injuries. Stem Cell Res Ther. 2015;6:173. doi: 10.1186/s13287-015-0172-4.

19. Constant CR, Murley AH. A clinical method of functional assessment of the shoulder. Clin Orthop Relat Res. 1987;(214):160-4.

20. Michener LA, McClure PW, Sennett BJ. American Shoulder and Elbow Surgeons Standardized Shoulder Assessment Form, 
patient self-report section: reliability, validity, and responsiveness. J Shoulder Elbow Surg. 2002;11(6):587-94. doi: 10.1067/mse.2002.127096.

21. Middleton KK, Barro V, Muller B, Terada S, Fu FH. Evaluation of the effects of platelet-rich plasma (PRP) therapy involved in the healing of sports-related soft tissue injuries. lowa Orthop J. 2012;32:150-63.

22. Koester MC, Dunn WR, Kuhn JE, Spindler KP. The efficacy of subacromial corticosteroid injection in the treatment of rotator cuff disease: a systematic review. J Am Acad Orthop Surg. 2007;15(1):3-11.

23. Kesikburun S, Tan AK, Yilmaz B, Yaşar E, Yazicioğlu K. Plateletrich plasma injections in the treatment of chronic rotator cuff tendinopathy: a randomized controlled trial with 1-year follow-up. Am J Sports Med. 2013;41(11):2609-16. doi: 10.1177/0363546513496542.

24. Rha DW, Park GY, Kim YK, Kim MT, Lee SC. Comparison of the therapeutic effects of ultrasound-guided platelet-rich plasma injection and dry needling in rotator cuff disease: a randomized controlled trial. Clin Rehabil. 2013;27(2):113-22. doi: 10.1177/0269215512448388.

25. Say F, Gurler D, Bulbul M. Platelet-rich plasma versus steroid injection for subacromial impingement syndrome. J Orthop Surg (Hong Kong). 2016;24(1):62-6. doi: 10.1177/ 230949901602400115.

26. Wesner M, Defreitas $\mathrm{T}$, Bredy $\mathrm{H}$, et al. A pilot study evaluating the effectiveness of platelet-rich plasma therapy for treating degenerative tendinopathies: a randomized control trial with synchronous observational cohort. PLoS One. 2016;11(2):e0147842. doi: 10.1371/journal.pone.0147842.

27. Jo CH, Lee SY, Yoon KS, Oh S, Shin S. Allogenic pure platelet-rich plasma therapy for rotator cuff disease: a bench and bed study. Am J Sports Med. 2018;46:3142-54. doi: 10.1177/0363546518800268.

28. Moraes VY, Lenza M, Tamaoki MJ, Faloppa F, Belloti JC. Platelet-rich therapies for musculoskeletal soft tissue injuries. Cochrane Database Syst Rev. 2014;(4):CD010071. doi: 10.1002/14651858.CD010071.pub3.

29. Fitzpatrick J, Bulsara M, Zheng MH. Effectiveness of PRP in the treatment of tendinopathy: response. Am J Sports Med. 2016;44(10):NP53. doi: 10.1177/0363546516669312.

30. Noh KC, Liu XN, Zhuan Z, et al. Leukocyte-poor platelet-rich plasma-derived growth factors enhance human fibroblast proliferation in vitro. Clin Orthop Surg. 2018;10(2):240-7. doi: 10.4055/cios.2018.10.2.240.

31. von Wehren L, Blanke F, Todorov A, Heisterbach P, Sailer J, Majewski M. The effect of subacromial injections of autolo- gous conditioned plasma versus cortisone for the treatment of symptomatic partial rotator cuff tears. Knee Surg Sports Traumatol Arthrosc. 2016;24(12):3787-92. doi: 10.1007/s00167015-3651-3.

32. Magalon J, Bausset $\mathrm{O}$, Serratrice N, et al. Characterization and comparison of 5 platelet-rich plasma preparations in a singledonor model. Arthroscopy. 2014;30(5):629-38. doi: 10.1016/ j.arthro.2014.02.020.

33. McCarrel TM, Minas T, Fortier LA. Optimization of leukocyte concentration in platelet-rich plasma for the treatment of tendinopathy. J Bone Joint Surg Am. 2012;94(19):e143(1-8). doi: 10.2106/JBJS.L.00019.

34. Anitua E, Zalduendo M, Troya M, Padilla S, Orive G. Leukocyte inclusion within a platelet rich plasma-derived fibrin scaffold stimulates a more pro-inflammatory environment and alters fibrin properties. PLoS One. 2015;10(3):e0121713. doi: 10.1371/journal.pone.0121713.

35. Cross JA, Cole BJ, Spatny KP, et al. Leukocyte-reduced plateletrich plasma normalizes matrix metabolism in torn human rotator cuff tendons. Am J Sports Med. 2015;43(12):2898-906. doi: 10.1177/0363546515608157.

36. Carmona JU, López C, Sandoval JA. Review of the currently available systems to obtain platelet related products to treat equine musculoskeletal injuries. Recent Pat Regen Med. 2013;3(2):148-59. doi: 10.2174/2210296511303020005.

37. DeLong JM, Russell RP, Mazzocca AD. Platelet-rich plasma: the PAW classification system. Arthroscopy. 2012;28(7):9981009. doi: 10.1016/j.arthro.2012.04.148.

38. Fitzpatrick J, Bulsara M, Zheng MH. The effectiveness of platelet-rich plasma in the treatment of tendinopathy: a metaanalysis of randomized controlled clinical trials. Am J Sports Med. 2017;45(1):226-33. doi: 10.1177/0363546516643716.

39. Stenhouse G, Sookur P, Watson M. Do blood growth factors offer additional benefit in refractory lateral epicondylitis? A prospective, randomized pilot trial of dry needling as a standalone procedure versus dry needling and autologous conditioned plasma. Skeletal Radiol. 2013;42(11):1515-20. doi: 10.1007/s00256-013-1691-7.

40. Yan R, Gu Y, Ran J, et al. Intratendon delivery of leukocytepoor platelet-rich plasma improves healing compared with leukocyte-rich platelet-rich plasma in a rabbit achilles tendinopathy model. Am J Sports Med. 2017;45(8):1909-20. doi: 10.1177/0363546517694357.

41. Lim W, Park SH, Kim B, Kang SW, Lee JW, Moon YL. Relationship of cytokine levels and clinical effect on platelet-rich plasma-treated lateral epicondylitis. J Orthop Res. 2018;36(3):91320. doi: 10.1002/jor.23714. 